International Journal of Computer Engineering and Technology (IJCET)

Volume 12, Issue 1, January-April 2021, pp. 1-10, Article ID: IJCET_12_01_001

Available online at https://iaeme.com/Home/issue/IJCET?Volume=12\&Issue $=1$

Journal Impact Factor (2020): 10.8630 (Calculated by GISI) www.jifactor.com

ISSN Print: 0976-6367 and ISSN Online: 0976-6375

DOI: 10.34218/IJCET.12.1.2021.001

(C) IAEME Publication

Scope Database Indexed

\title{
SYSTEMATIC REVIEW OF TECHNOLOGIES USED FOR AUTOMATIC CARICATURE GENERATION
}

\author{
Priya Shah, Rajasi Adurkar, Shreya Desai, Swapnil Kadakia and Chetashri Bhadane \\ Department of Computer Engineering, Dwarkadas J. Sanghvi College of Engineering, \\ Mumbai, Maharashtra, India
}

\begin{abstract}
Definition -Cartoons have been in the industry since the middle ages and are now emerging into an upgraded version of themselves, widely referred to as caricatures. Caricatures are mainly used to interpolate humor and satire in advertisements or comics. A gradual shift from cartoons to caricatures took place mainly due to the liveliness and realism of the representations generated. In the initial years of transformation into this newly developed area, artists used to manually draw the caricatures. However, with the advent of digitalization, in a short time span, various software was launched in the market to automate the process. They did save a lot of time, but the quality was hampered. Thus, the main objective of this paper is to present a comparative study of different methodologies to determine and implement the best model for automatic caricature generation that will not only save time but also consider various aspects of facial representation.
\end{abstract}

Key words: caricature generation, deep learning, generative adversarial networks, neural networks, literature review

Cite this Article: Priya Shah, Rajasi Adurkar, Shreya Desai, Swapnil Kadakia and Chetashri Bhadane, Systematic Review of Technologies used for Automatic Caricature Generation, International Journal of Computer Engineering and Technology 12(1), 2021, pp. 1-10.

https://iaeme.com/Home/issue/IJCET?Volume=12\&Issue=1

\section{INTRODUCTION}

Caricatures are facial portraits by artists that exaggerate only a few distinctive facial features. It is a type of visual communication that carries the message with overstated drawings. These exaggerations performed cause maximum deformation of the features and yet the caricatures are still recognizable by humans [10]. A caricature is certainly defined as "a photo, description, or imitation of an individual or a thing that maintains the striking characteristics through the exaggeration procedure so as to give a comic or grotesque effect." Any subject which concerns 
humanity can be subject to caricature. With contemporaneousness, the subject has become as important as the object. As an art branch of modernism, the caricature has become a medium, which all the antagonism, and mutiny found a voice in. While the aim of art has been mentioned repeatedly, a caricature is transient and clear. Its goal is to wake individuals up with a sledgehammer; and it is very thriving in reaching its goal. It does not concern to be like or dislikable, it is terribly sincere in its manner [11]. With the emergence of deep learning, recognition performances by computers on real-world faces became like human performance even below dissipated situations. However, there is still a spot in caricature recognition performance between computers and humans.

Today, in the Claymation industry, time is one of the most vital aspects because it is the only thing that is truly finite. Pertaining to that, on average it takes up about a day to complete a comic book page, if opted for free-hand, according to a few tweets of artists talking about their efforts to complete pages [12]. However, with the new state of the art technologies, facts supported by evidence convey that the time required to complete a comic strip can be reduced to nearly half. Therefore, there is an ardent need in this industry to automate the caricature generation process. It is impossible to replace the basic essence of caricature generation i.e., imagination. A computer to date has not been able to function in exactly the same way a human brain does. Nonetheless, the style of a caricature artist can be studied and learned to create similar, if not identical, caricatures.

In this paper, the main aim is to perform a systematic review of the different methods available to automate the caricature generation process. The paper compares the different technologies available along with the efficiency and accuracy of the different methodologies present to insinuate the one model that can give the best results.

Section 2 provides the comparative review methodology used to screen the different papers available in IEEE, Springer and other such journals. Section 43 mentions the findings and results after the survey, Section 4 demonstrates the implementation followed by Section 5 which depicts the results obtained. Finally, Section 6 gives a conclusion.

\section{COMPARATIVE REVIEW METHODOLOGY}

A systematic review, or systematic literature review, is a kind of literature review that utilizes structured methods to compile subsidiary data, critically evaluate research studies, and integrate findings qualitatively or quantitatively [14]. metrics, design metrics, code related metrics, testing metrics. All these are used by management to derive new metrics to check the health of the project.

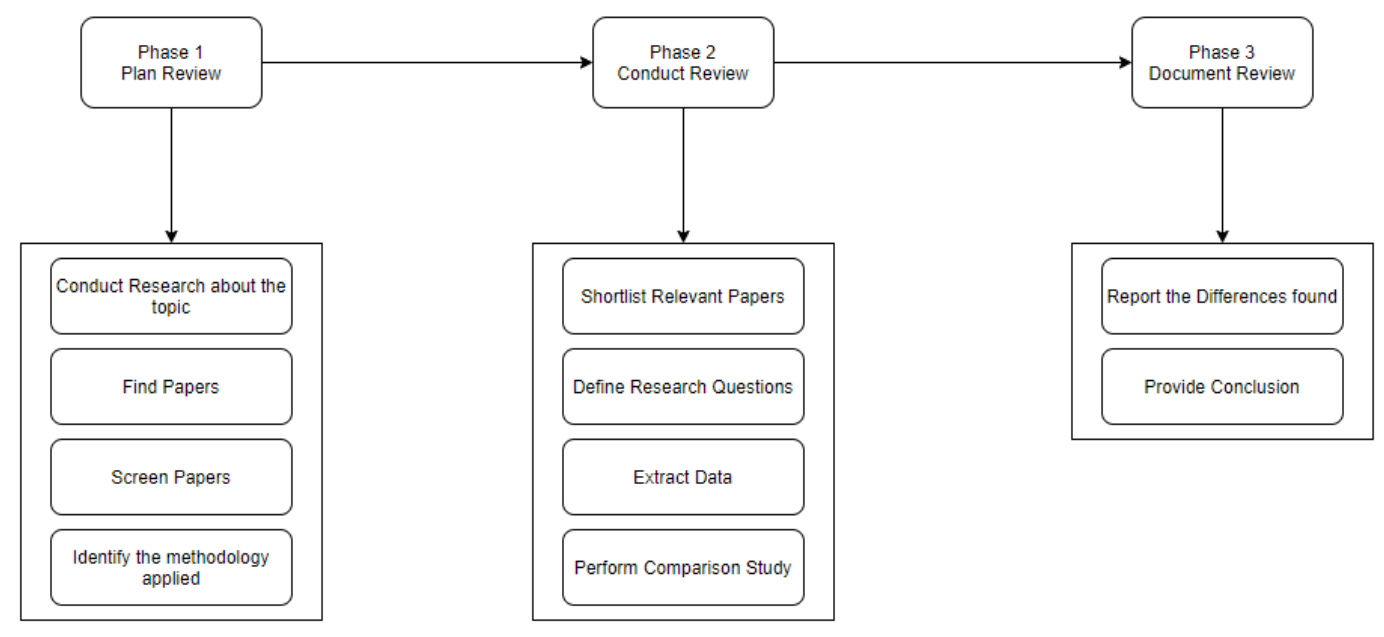

Figure 1 Impact of Process Models on Various Factors 
The main source of papers was from renowned digital libraries like IEEE Xplore, arXiv, Springer, ACM, Science Direct. A lot of papers were screened and those that did not have English as the main language, had not full text availability and had outdated technologies were eliminated. The nine published papers that proposed novel methods and focused solely on caricature generation as the primary domain along with accurate results were selected.

\section{INFORMATION EXTRACTION}

\subsection{What are the Different Approaches Adopted for Generating Caricature?}

With significant progress in the field of computer graphics and neural networks, the recreation of art by a computer has become feasible with adequate resemblance to the original artistic styles and techniques. Different procedures employed for generating caricatures yield different results and hence a comprehensive review of the different methodologies should be done before implementing the same. Generation of caricatures from a portrait consists primarily of the following two systems:- Geometric exaggeration and Texture/Style transfer. Selecting a methodology that strikes the correct balance between the above-mentioned operations is essential. Works like [1] and [2] mainly concentrate on the geometric face deformations. In [1], since the main focus is geometric deformation, a sequence-to-sequence neural network model is proposed to extract the explicit facial features. The encoder is a bi-directional RNN and the decoder is an autoregressive RNN. Further, thin-plate spline (TPS) transformation is used for the exaggeration and deformation. Unpaired photo-to-caricature translation was proposed in, CariGAN [4], with the use of Generative Adversarial Network (GAN). Two separate modules, namely, CariStyGAN and CariGeoGAN were proposed for appearance stylization and geometric exaggeration respectively to make the process more robust. As opposed to the previous methodology texture transfer was a vital factor in this approach. Another approach [3] was to employ a 3D morphable mesh model or 3DMM model (Surrey Face model) and use a 'measure globally, weight locally' technique to deform the original facial structure. The facial landmarks were automatically detected and with the help of 3DMM a 3D face structure of the image was constructed. Further, the divergence of the facial regions was determined by comparing the 3D structure with a reference model. These altered regions were then used for deforming the original mesh, thereby obtaining the 2D caricature in the original camera view. The main aim of this work was to prove that caricatures find an important application in facial recognition. In this paper [9], a novel technique is being proposed to generate three dimensional caricatures automatically from two dimensional photos. In addition to generating $2 \mathrm{D}$ caricatures from 2D photos, construction of 3D caricatures is also required. The process employed for generating 3D caricatures comprises mainly of the following three steps: (1) Generation of 3D caricature meshes from the 2D photos, (2) Transfer of photos to 2D caricatures utilized for texture images, and (3) Reconstruction of a 3D face similar to the 3D caricature mesh created in step (1). They both have the same mesh connectivity which ensures a direct transfer of texture coordinates between the 3D face and generated 3D caricature mesh.

\subsection{Identifying the Datasets and Attributes Used for Training and Evaluating Models}

Datasets are elementary to stimulate the advancement of several data-processing fields, giving scope, validity, and assurance to results. Thus, it is crucial to review the dataset and the attributes it contains to get a better understanding of the efficiency and accuracy of the model used to generate caricatures. Many papers have created their dataset. One dataset created [1] included 39-person portrait photos and their corresponding caricatures, which is 503 pairs of images and a total of 1006 images. It consisted of 68 feature points as attributes. [2] mainly concerns the size, length, and structure of the face. With an improved ASM method, it extracts 
144 facial feature points from the face image. [3] has used AFLW, IJB-A, VGG, LFW, and MegaFace datasets and 68 landmark format as attributes. [4] uses an unpaired dataset. For the domain of photos, they have used the CelebAMask-HQ dataset [5] which contains 30, 000 portrait photos and for the domain of 3D caricatures, they created their 3DCari dataset which contains 6, 100 3D caricature meshes with the same topology.

\subsection{Identifying the Research Gaps}

In the future, [1] suggests the expansion of their dataset study on how to particularly handle definitely more facial details during the stylization operation, and make basically stylized literally faces definitely more closely, which basically is quite significant. Furthermore, it proposes some end-to-end network to directly generate portrait caricature.[2] will particularly improve their work and for all intents and purposes do some particularly further research in the following aspects:

- Study more creation styles of different animators to literally improve the existing algorithms and make fairly more kinds of exaggeration rules to for the most part get various effects with a different style in a subtle way.

- Study image morphing methods fairly further to for the most part improve the morphing speed and basically enhance the control and quality of image morphing in a fairly big way.

- Try generally more texture effects when generating portraits with exaggeration besides sketch style in a kind of major way.

- suggests researching more on caricature to photo translation and also purposes video caricature for future.

\subsection{Comparison of Distinct Algorithms Selected}

Algorithms in Machine Learning are the ones that learn from the training data and then display results on unseen data. In the formation of GANs, due to the presence of multiple factors, various algorithms are used to get the best possible results on each of the factors. [1] uses basic algorithms namely RNN(LSTM) for the encoder module whereas the Auto-regressive RNN(HyperLTSM) for the decoder module which work mainly on geometrical and structural features. To further enhance the results, Adam algorithm is used for optimization and backward propagation. Later, [2] used real faces as input using the Algorithm of Facial Form Exaggeration and The Discovery of Obvious Features of Five Sense Organs and its Exaggeration to produce slightly better results as compared to [1]. [4] produced results with primary focus on appearance using PCA with automatic landmark detector and image warping for synthesis. [13] consisting of style network uses MUNIT and geometric network which further utilizes augmented CycleGAN and LSGAN and the identity preservation algorithm with the help of loss functions. To venture out into $3 \mathrm{D}$, [3] devices a technique to improve face recognition using $\mathrm{A}$ combination of multiple landmark localization algorithms, Laplacian mesh editing algorithm and CNN. Finally, [9] creates 3D caricatures using 3D caricature meshes from 2D photos with the help of consistent projection of landmark positions. 


\subsection{Determining the Loss Functions Selected for Enhancing the Result}

Table 1 A comparative study of different Loss Functions studied

\begin{tabular}{|c|c|}
\hline [1] & $\begin{array}{l}\text { This paper uses a traditional reconstruction loss function and matching loss function. } \\
\text { These } 2 \text { loss functions are optimized separately and differently. The matching loss } \\
\text { handles the similarity of images that belong to the same individual. While training } \\
\text { the model, the weights of the encoder are optimized using the minibatch and then the } \\
\text { weights are kept frozen. Finally, the weights of the decoder are optimized with the } \\
\text { help of SGD with RMSprop. }\end{array}$ \\
\hline [4] & $\begin{array}{l}\text { There are three types of loss defined in the CariGeoGAN, a CycleGAN inspired } \\
\text { architecture. } \\
\text { Firstly, adversarial loss, a broadly used loss in GANs, is incorporated. In order to } \\
\text { foster the indistinguishable generating landmark from the physically drawn } \\
\text { caricatured images, this loss is applied along with the model. Additionally, this loss } \\
\text { is used to support the generation of portrait photo landmarks, which are not } \\
\text { distinguished by the adversary. } \\
\text { Secondly, bidirectional cycle-consistency loss is used, which is applied with } \\
\text { CycleGAN to put a constraint in the cycle consistency between the forward mapping } \\
\text { and the backward mapping. It further helps to limit the mapping solution from giving } \\
\text { the input to obtaining output. Though this loss is not highly guaranteed. } \\
\text { Finally, a characteristic loss was included to penalize the cosine differences, after } \\
\text { removing the corresponding means, between the predicted and the input landmark. } \\
\text { It makes the exaggeration meaningful as it captures the most distinctive feature as } \\
\text { compared with the mean face and then exaggeration of facial features is performed. }\end{array}$ \\
\hline [3] & $\begin{array}{l}\text { By minimizing the loss function here, it aids in deciding the landmarks of the method } \\
\text { with maximum likelihood. Once the probe image is given as input with the respective } \\
\text { landmarks of the landmark localization methods, the part of the loss function } \\
\text { provides the likelihood of each method, the one with maximum likelihood is selected. }\end{array}$ \\
\hline [9] & $\begin{array}{l}\text { The paper includes adversarial loss, cycle-consistency loss, character loss and user } \\
\text { loss. These four are successively added to the objective function. } \\
\text { First of all, the adversarial loss ensures that the results generated are 3D, } \\
\text { nevertheless, they are highly random. } \\
\text { The second loss function, cycle-consistency, regularizes the mapping to make the } \\
\text { results less extreme but fails to retain identity and expression. } \\
\text { The third one, character loss, constraints to have the same identity and expression for } \\
\text { both the input and the generated result. Lastly, the user loss keeps a track of details } \\
\text { and handles only the proportion of facial shapes. }\end{array}$ \\
\hline [13] & $\begin{array}{l}\text { Here while training the model, the following types of loss functions were minimized: } \\
\text { 1. The reconstruction loss of the image, the style latent code and the landmark } \\
\text { transformation latent code, and the cycle consistency loss of the content code and of } \\
\text { the image. } \\
\text { 2. The generative adversarial loss pairs on images, landmarks and latent codes. } \\
\text { 3. The identity loss on the image and on the landmark. }\end{array}$ \\
\hline
\end{tabular}

\section{IMPLEMENTATION}

A significant amount of work has been done by many people in an effort to generate computerized caricatures using the different learning methodologies in deep learning. Works in automatic caricature generation can be roughly categorized into three parts - geometric shape exaggeration, texture rendering, or both. Earlier works [15] [16] have tried to either work in 
geometric feature exaggeration or rendering of texture styles thereby preserving the identity of the original image. After considering all the factors and performing a systematic review, it can be concluded that WarpGAN [6] covers the majority of the aspects of caricature generation and can produce outputs which closely resemble the original input.

In this work, the following aspects of caricature generation are being tackled:

- Warping the face photo to exaggerate certain geometrical features of the face to give it a grotesque effect.

- Rendering of the texture styles into the deformed face to decouple the style transfer process from that of the shape deformation one.

- Taking into consideration the identity preservation loss and reconstruction loss to preserve the identity of the original photo.

\subsection{Dataset}

The dataset used in this project [7] [8] is a large photograph-caricature dataset which consists of 5,974 photographs and 6,042 caricatures from 252 people. 17 labeled facial landmarks are provided for each image in the dataset, however, all the images with 5 landmarks are aligned. Further, the images are aligned through similarity transformation using the five landmarks and resized to $256 \times 256$.

The dataset is randomly split into:

- Training dataset - Contains 126 identities which consist of 3,016 photos and 3,112 caricatures.

- Testing dataset - Contains 126 identities which consist of 2,958 photos and 2,930 caricatures.

\subsection{Working}

The WarpGAN [6] is mainly implemented in 2 phases:

\subsubsection{Generator}

The deformable generator in WarpGAN is comprised of three sub-networks, namely - a content encoder $\left(E_{c}\right)$ to output a feature map $E_{c}(x)$, a decoder $(R)$ to render an image having a particular style and a warp controller to warp the rendered image.

An image $x_{c}$ is given as input to the content encoder from the domain of face photos $X_{p}$. The output obtained is a feature map $E_{c}(x)$. This feature map in addition to the random latent style code $s$, produced by the style encoder $\left(E_{S}\right)$, is given as an input to the decoder. An unsupervised method is chosen to detach the style representation from the feature map. This ensures a wide variety of texture styles can be transferred in the input photo, as present in the caricature domain. Further, the decoder renders an image having a specific style. In order to control the visual styles, the decoder $(R)$ applies a multi-layer perceptron to generate the parameters of the Adaptive Instance Normalization (AdaIN) layers. $E_{c}$ and $(R)$ are regularized to preserve semantic information, by combining the Identity mapping loss and the Adversarial Loss.

Finally, the warp controller is responsible for evaluating the control points, as well as their displacements to warp the image. The warp controller is a sub-network of two-fully connected layers. Latent Feature map $E_{c}(x)$ is given as an input to the warp controller. It estimates $\mathrm{k}$ control points $\mathrm{p}=\left\{\mathrm{p}_{1}, \mathrm{p}_{2}, \mathrm{p}_{3}, \ldots \ldots ., \mathrm{p}_{\mathrm{k}}\right\}$ and their corresponding displacement vector.

$\Delta \mathrm{p}=\left\{\Delta \mathrm{p}_{1}, \Delta \mathrm{p}_{2}, \Delta \mathrm{p}_{3}, \ldots \ldots ., \Delta \mathrm{p}_{\mathrm{k}}\right\}$. These points are then fed into the differentiable warping module. Let $\mathrm{p}^{\prime}=\left\{\mathrm{p}_{1}^{\prime}, \mathrm{p}_{2}{ }_{2}, \mathrm{p}_{3}^{\prime}, \ldots \ldots . ., \mathrm{p}_{\mathrm{k}}\right\}$ be the destination points, where $\mathrm{p}_{\mathrm{i}}^{\prime}=\mathrm{p}_{\mathrm{i}}+\Delta \mathrm{p}_{\mathrm{i}}$. Further, the grid sampler of size $H \times W$ is computed via thin-plate spline interpolation. 


$$
f(q)=\sum_{i=1}^{k} \omega_{i} \varphi\left(\left\|q-p_{i}^{\prime}\right\|\right)+v^{T} q+b
$$

Here, the vector $q$ denoted the $u-v$ location of a pixel in the resultant image, and $f(q)$ gives the inverse mapping of $\mathrm{q}$ in the original image. $\varphi\left(\left\|\mathrm{q}-\mathrm{p}_{\mathrm{i}}^{\prime}\right\|\right)$ is the kernel function. $\mathrm{W}, \mathrm{v}, \mathrm{b}$ are the parameters that are fitted to minimize the $\sum_{i=1}^{k}\left\|\mathrm{f}\left(\mathrm{p}_{\mathrm{i}}^{\prime}\right)-\mathrm{p}_{\mathrm{j}}\right\|^{2}$ and a curvature constraint which can be solved in closed form. The warped image $\mathrm{G}(\mathrm{x}, \mathrm{s})=\operatorname{Warp}\left(\mathrm{R}\left(\mathrm{E}_{\mathrm{c}}(\mathrm{x}), \mathrm{s}\right), \mathrm{p}, \Delta \mathrm{p}\right)$ can then be generated through bi-linear sampling.

\subsubsection{Discriminator}

It comprises the Patch Adversarial Loss and Identity-Preservation Adversarial Loss. Initially a fully Convolutional network is used as a patch discriminator. The patch discriminator is trained as a 3-class classifier to enlarge the difference between the types of generated pictures and real photos. Let D1, D2, and D3 denote the logits for the three categories of caricatures, photos, and generated pictures, respectively. The patch adversarial loss is stated as follows:

$$
\begin{gathered}
L_{p}^{G}=-E_{x_{p} \in X_{p}, s \in S}\left[\log D_{1}\left(G\left(x_{p}, s\right)\right)\right] \\
L_{p}^{D}=-E_{x_{c} \in X_{c}}\left[\log D_{1}\left(x_{c}\right)\right]-E_{x_{p} \in X_{p}}\left[\log D_{2}\left(x_{p}\right)\right]-E_{x_{p} \in X_{p}, s \in S}\left[\log D_{3}\left(G\left(x_{p}, s\right)\right)\right]
\end{gathered}
$$

Though patch discriminator is appropriate for learning visual style transfer and classifies the particular patch as real or fake, it fails to capture the identifying features of various identities. The exaggeration designs for various folks might actually diverge and is according to their facial expressions. To mix the identity-preservation and identity specific style learning, this methodology tends to propose to prepare the discriminator as a $3 \mathrm{M}$-class classifier, wherever $\mathrm{M}$ refers to identities. The first $\mathrm{M}$ class corresponds to different identities real photos, second corresponds to different identities real caricatures, and third $\mathrm{M}$ class corresponds to different identities of real photos and fake caricatures.

Now, consider $\mathrm{y}^{\mathrm{p}}, \mathrm{y}^{\mathrm{c}} \in\{1,2,3, \ldots \mathrm{M}\}$ as identity labels of the photos and caricatures, respectively. The identity-preservation adversarial losses for generator $G$ and discriminator $D$ are as follow:

$$
\begin{aligned}
L_{p}^{G}=-E_{x_{p} \in X_{p}, s \in S} & {\left[\log D\left(y_{p} ; G\left(x_{p}, s\right)\right)\right] } \\
L_{p}^{D}=-E_{x_{c} \in X_{c}} & {\left[\log D\left(y_{c} ; x_{c}\right)\right]-E_{x_{p} \in X_{p}}\left[\log D\left(y_{p}+M ; x_{p}\right)\right] } \\
& -E_{x_{p} \in X_{p}, s \in S}\left[\log D\left(y_{p}+2 M ;\left(x_{p}, s\right)\right)\right]
\end{aligned}
$$

Here, $\mathrm{D}(\mathrm{y} ; \mathrm{x})$ denotes the logits of classy given an image $\mathrm{x}$. It is trained well to distinguish between real photos, real caricatures, generated caricatures as well as the identities in the photo. The training of the generator is done to fool the discriminator in recognizing the generated image as a true caricature of the corresponding identity. Finally, the system is optimized in associate degree end-to-end approach with the subsequent objective functions:

$$
\begin{gathered}
\min _{G} L_{G}=\lambda_{p} L_{g}^{G}+\lambda_{g} L_{g}^{G}+\lambda_{\text {idt }}\left(L_{i d t}^{c}+L_{i d t}^{P}\right) \\
\min _{D} L_{D}=\lambda_{p} L_{g}^{D}+\lambda_{g} L_{g}^{D}
\end{gathered}
$$

For comparing the variations in the given module, the losses are removed individually. From the results, when an identity-preservation adversarial loss is removed, it can be derived that the discriminator fails to focus on personal styles and features leading to randomness in the geometry of the facial texture. Secondly, on removing the patch adversarial loss, the focus of the discriminator shifts to geometry and facial shape only. This leads to a negative impact on the output since textures styles are predominant in caricature generation which are overlooked by the above mentioned two losses. On the contrary, it is observed that when identity mapping 
loss is removed, the model still performs much better than the rest of the cases. And hence it is kept for image quality enhancement.

While creating caricatures, one of the vital aspects is identity preservation, the constraint to which is included in the adversarial loss. This will not only help the network in generating detailed facial representations but also improve the uniqueness with variety in distinct features. The highest priority is assigned to perceptible generation because more recognizable features will be the accuracy in facial recognition which can be implemented in the future.

Mostly machine learning algorithms are deterministic models. This model however provides the flexibility to the users to customize the exaggeration by introducing a parameter ' $\alpha$ '. The extent of shape exaggeration will be controlled by $\alpha$ before the image warping takes place. The results will differ based on the input values of $\alpha$. When $\alpha={ }^{\prime} 0$ ', only the texture is changed. When $\alpha={ }^{\prime} 1$ ', it results in the original output generated after image warping. And lastly when $\alpha=$ ' 2 ', features are exaggerated. $\alpha$ accepts values up to 1 decimal place resulting in the appropriate exaggeration of the facial representation.

\section{RESULTS}

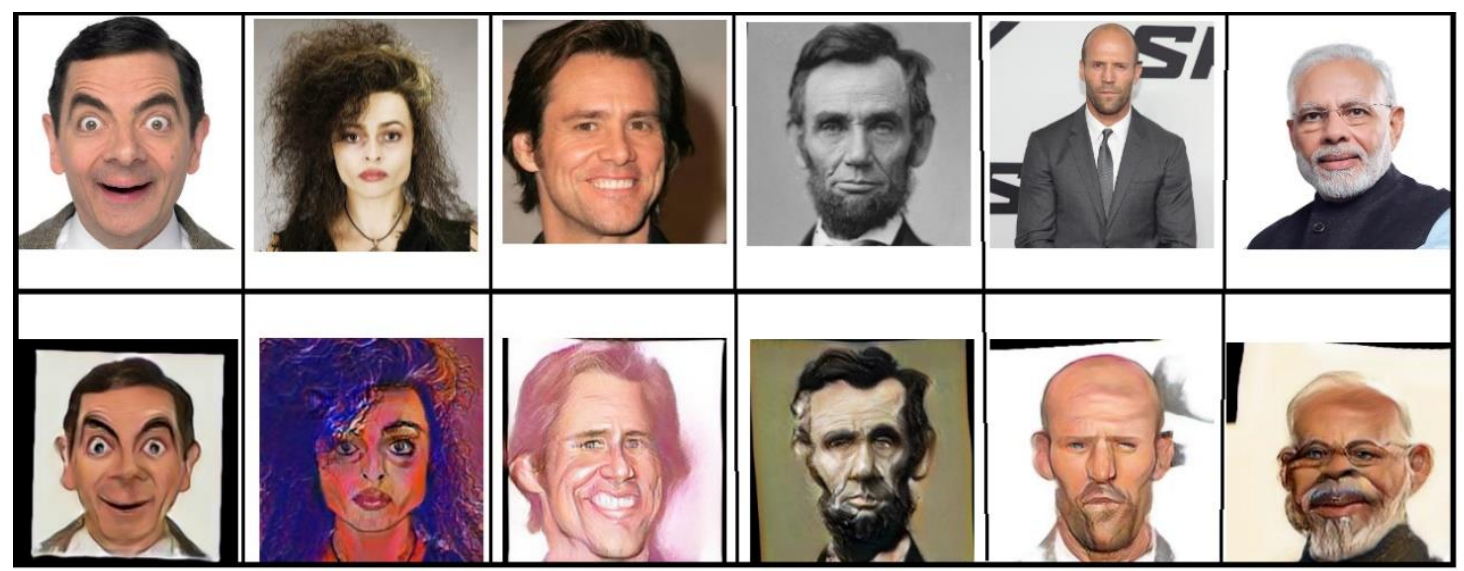

Figure 2 Depicts the results obtained from WarpGAN. First row shows the hand-drawn caricatures, while the second row shows the images generated as the output.

\section{CONCLUSION}

Caricatures are a fundamental form of art, and their generation cannot be truly replaced by computers. Nevertheless, efforts need to be made in order to conserve time by making the above process digitized. Content creation requires the rapid production of illustrations at the cost of quality. As a result, the automatic generation of such imagery saves a lot of time and work. Speedy recovery of images back from the caricatures make the automatic generation of them a vital need of the industry.

With significant progress in the field of computer graphics and neural networks, the recreation of art by a computer has become feasible with adequate resemblance to the original artistic styles and techniques. Even so, in some cases, there may be a possibility of missing out on a few salient features due to constraints on the warping handles leading to weaker standalone warps.

Different procedures employed for generating caricatures yield different results and hence a comprehensive review of the different methodologies should be done before implementing the same. In this paper, a comparison of multiple papers based on different factors like approaches, datasets, research gaps and algorithms is conducted and a comprehensive study is carried out to determine what technique best generates a caricature that performs geometric 
exaggeration as well as texture rendering and also incorporates a good resemblance of the subject.

\section{REFERENCES}

[1] K. Xu, C. Tang, J. Lv and Z. He, "Exaggerated Portrait Caricatures Generation Based on Seq2Seq," 2019 9th International Conference on Information Science and Technology (ICIST), Hulunbuir, China, 2019, pp. 36-44, doi: 10.1109/ICIST.2019.8836944.IEEE Std 610.12-1990: IEEE standard glossary of software engineering terminology, 1990

[2] C. Li and Z. Miao, "A Method for Exaggerative Caricature Generation from Real Face Image," 2013 2nd IAPR Asian Conference on Pattern Recognition, Naha, 2013, pp. 828832, doi: 10.1109/ACPR.2013.150.

[3] J. Neves and H. Proença, "“A Leopard Cannot Change Its Spots": Improving Face Recognition Using 3D-Based Caricatures," in IEEE Transactions on Information Forensics and Security, vol. 14, no. 1, pp. 151-161, Jan. 2019, doi: 10.1109/TIFS.2018.2846617.

[4] K. Cao, J. Liao, and L. Yuan, "CariGANs: Unpaired Photo-to-Caricature Translation, " in ACM Transactions on Graphics, Vol. 37, No. 6, Article 244., November 2018, doi: $10.1145 / 3272127.3275046$

[5] Lee, C.H., Liu, Z., Wu, L., Luo, P.: Maskgan: Towards diverse and interactive facial image manipulation. arXiv preprint arXiv:1907.11922 (2019) Humphrey, W. Introduction to Personal Software Process. Addison-Wesley, 1997.

[6] Y. Shi, D. Deb and A. K. Jain, "WarpGAN: Automatic Caricature Generation," 2019 IEEE/CVF Conference on Computer Vision and Pattern Recognition (CVPR), Long Beach, CA, USA, 2019, pp. 10754-10763, doi: 10.1109/CVPR.2019.01102.

[7] J. Huo, W. Li, Y. Shi, Y. Gao and H. Yin, "WebCaricature: a benchmark for caricature recognition," British Machine Vision Conference, 2018, arXiv:1703.03230

[8] J. Huo, Y. Gao, Y. Shi, and H. Yin, "Variation Robust Cross-Modal Metric Learning for Caricature Recognition," In Proceedings of the Thematic Workshops of ACM Multimedia 2017 (Thematic Workshops '17). Association for Computing Machinery, New York, NY, USA, 340-348. DOI:https://doi.org/10.1145/3126686.3126736

[9] Zipeng Ye, Ran Yi, Minjing Yu, Juyong Zhang, Yu-Kun Lai, Yong-Jin Liu, "3D-CariGAN: An End-to-End Solution to 3D Caricature Generation from Face Photos", 2020/03/15.

[10] The Significance of Caricature in Visual Communication Baran Sarıül Course Coordinator: Alessandro Segalini Izmir University of Economics, Faculty of Fine Arts and Design Department of Visual Communication Design Spring 2009, Gd494

[11] "On the Origin of Sketches: The Evolution of Technology for ...." 10 Feb. 2020, https://www.gravitysketch.com/blog/articles/on-the-origin-of-sketches-the-evolution-oftechnology-for-ideation/. Accessed 30 Apr. 2020.

[12] "How long does it take normally for a cartoonist to finish one...." 24 Dec. 2014, https://www.quora.com/How-long-does-it-take-normally-for-a-cartoonist-to-finish-onepage-of-a-comic-drawing-eg-Marvel-comic. Accessed 30 Apr. 2020.

[13] https://arxiv.org/pdf/2001.01870v1.pdf 
[14] Armstrong R, Hall BJ, Doyle J, Waters E (March 2011). "Cochrane Update. 'Scoping the scope' of a cochrane review". Journal of Public Health. 33 (1): 147-50. doi:10.1093/pubmed/fdr015. PMID 21345890.

[15] Y. Alharbi, N. Smith and P. Wonka, "Latent Filter Scaling for Multimodal Unsupervised image-to-image Translation," 2019 IEEE/CVF Conference on Computer Vision and Pattern Recognition (CVPR), Long Beach, CA, USA, 2019, pp. 1458-1466, doi: 10.1109/CVPR.2019.00155.

[16] J. Zhu, T. Park, P. Isola and A. A. Efros, "Unpaired Image-to-Image Translation Using Cycle-Consistent Adversarial Networks," 2017 IEEE International Conference on Computer Vision (ICCV), Venice, 2017, pp. 2242-2251, doi: 10.1109/ICCV.2017.244. 